\title{
Collider limits on new physics within micrOMEGAs_4.3
}

\author{
D. Barducci ${ }^{1 *}$, G. Bélanger ${ }^{1 \dagger}$, J. Bernon ${ }^{2 \ddagger}$, F. Boudjema ${ }^{1 \S}$, J. Da Silva ${ }^{1,2 \pi}$, \\ S. Kraml ${ }^{2 \|}$, U. Laa ${ }^{1,2 * *}$, A. Pukhov ${ }^{3 \dagger \dagger}$, \\ ${ }^{1}$ LAPTH, Université Savoie Mont Blanc, CNRS, B.P.110, \\ F-74941 Annecy-le-Vieux Cedex, France \\ ${ }^{2}$ Laboratoire de Physique Subatomique et de Cosmologie, Université Grenoble-Alpes, \\ CNRS/IN2P3, 53 Avenue des Martyrs, F-38026 Grenoble, France \\ ${ }^{3}$ Skobeltsyn Institute of Nuclear Physics, Moscow State University, \\ Moscow 119992, Russia
}

\begin{abstract}
Results from the LHC put severe constraints on models of new physics. This includes constraints on the Higgs sector from the precise measurement of the mass and couplings of the $125 \mathrm{GeV}$ Higgs boson, as well as limits from searches for other new particles. We present the procedure to use these constraints in micrOMEGAs by interfacing it to the external codes Lilith, HiggsSignals, HiggsBounds and SModels. A few dedicated modules are also provided. With these new features, micrOMEGAs_4.3.4 provides a generic framework for evaluating dark matter observables together with collider and non-collider constraints.
\end{abstract}

\footnotetext{
*Email: barducci@lapth.cnrs.fr

†Email: belanger@lapth.cnrs.fr

${ }^{\ddagger}$ Email: bernon@lpsc.in2p3.fr

$\S$ Email: boudjema@lapth.cnrs.fr

『Email: jonathan.dasilva@lapth.cnrs.fr

"Email: sabine.kraml@lpsc.in2p3.fr

**Email: ursula.laa@lpsc.in2p3.fr

††Email: pukhov@lapth.cnrs.fr
} 


\section{Contents}

1 Introduction 1

2 Higgs-sector constraints 2

2.1 Lilith . . . . . . . . . . . . . . . . . . . . 2

2.2 HiggsBounds and HiggsSignals . . . . . . . . . . . . . . . . . . . . 5

2.3 Automatic generation of interface files . . . . . . . . . . 7

3 Collider limits on new particles 8

3.1 LEP limits . . . . . . . . . . . . . . . . . . . . . . . . . 8

3.2 LHC limits from SModelS . . . . . . . . . . . . . . . . . . . 9

3.2 .1 Input files . . . . . . . . . . . . . . . . . 9

3.2 .2 Output format . . . . . . . . . . . . . . . . . 11

3.2 .3 Identification of the SM-like Higgs . . . . . . . . . . . . . . . . 13

3.2 .4 SMS caveats . . . . . . . . . . . . . . . . . 13

3.3 Other simplified-model limits . . . . . . . . . . . . . . . . . . 14

3.3.1 $Z^{\prime}$ searches . . . . . . . . . . . . . . . . . 14

3.3.2 Mono-jet searches . . . . . . . . . . . . . . . . 15

4 Installation and sample output $\quad 15$

5 Conclusions 17

6 Acknowledgements 18

\section{Introduction}

A plethora of particle dark matter candidates have been proposed in the last decades to explain the astrophysical and cosmological observations via extensions of the Standard Model (SM). In particular, new weakly interacting particles have attracted a lot of attention as natural cold dark matter candidates, see e.g. $[1,2]$ for comprehensive reviews. The list ranges from the well studied neutralino in the Minimal Supersymmetric Standard Model (MSSM) [3,4] to new fermionic or scalar states in a dark sector which communicates with the SM through some portal interactions [5,6]. The code micrOMEGAs [7-12] was developed to compute the properties and signatures of a dark matter candidate in a generic model of new physics. Furthermore, through its link to CalcHEP [13,14], observables at colliders can be computed. The code is widely used to compute dark matter observables and constraints in extensions of the SM.

With the extremely successful operation of the LHC and its experiments, which so far has led to the discovery of the Higgs boson $[15,16]$ and to a large number of constraints on the signals of other new particles (including dark matter) in a vast variety of channels [1721], checking the compatibility of a dark matter model with experimental observations is becoming an increasingly complex and time consuming task. This is especially true in the framework of new physics models with a number of new particles and signatures at the TeV scale. Dedicated public codes have been developed to meet this challenge: Lilith [22], HiggsSignals [23] and HiggsBounds [24,25] to check Higgs sector constraints, as well 
as CheckMate [26,27], MadAnalysis 5 [28,29], SModelS [30-32], Fastlim [33], XQCAT [34] and SUSY-AI [35] for testing the limits from searches for supersymmetry (SUSY) or some other new physics. It is the philosophy of micrOMEGAs to rely on other public codes when relevant. This has the advantage of providing a well-tested framework without duplicating work. We have therefore designed interfaces to some of the above-mentioned codes to confront the predictions of dark matter models to the LHC results.

In this paper we describe the facilities provided in micrOMEGAs 4.3 to check the collider constraints on the dark matter models provided with the public distribution. These range from in-house routines designed for imposing LEP constraints or limits on a new $Z^{\prime}$ at the LHC to interfaces to more complete codes that incorporate limits derived at the LHC Run 1. These include Lilith [22] and HiggsSignals [23] that check the compatibility to measurements of the Higgs boson at $125 \mathrm{GeV}$, HiggsBounds [24, 25] that provides modelindependent limits on additional neutral and charged Higgs states, and SModels [30-32] that checks the LHC limits on the production of new odd particles in the context of Simplified Model Spectra (SMS) constraints. For each of these facilities, we explain the functions that are provided in micrOMEGAs to impose constraints, their input format and the interpretation of the output.

The codes chosen for the interface with micrOMEGAs are not only fast and therefore suitable for large scans of parameter space, they are also quite generic and thus applicable to a wide range of models. Of course some limitations apply. For example, the Higgs signal strength approach in Lilith and HiggsSignals is not adapted for models where new structures in the Higgs vertices or new Higgs production modes appear. Moreover, the SMS results used in SModelS might not always be directly applicable to cases with a different spin structure than considered by the experiments. The user is advised to keep these limitations in mind for the proper usage of these codes in micrOMEGAs.

The paper is organised as follows. Section 2 deals with the tools for testing the Higgs sector: Lilith, HiggsSignals and HiggsBounds. Section 3 describes the interface to SModelS as well as the routines to test LEP limits and the routine to constrain a $Z^{\prime}$. An outlook for a new routine evaluating constraints from monojet searches is also given. Installation instructions as well as a sample output are given in Section 4. Section 5 contains our conclusions.

\section{Higgs-sector constraints}

Constraints originating from the measurements of signal strengths of the $125 \mathrm{GeV}$ state at LHC can be obtained by means of Lilith [22] or HiggsSignals [23] while constraints on additional Higgs states are accessible through HiggsBounds [25].

\section{$2.1 \quad$ Lilith}

Lilith [22] is a light and very fast Python library that can be used to derive constraints on the parameter space of new physics scenarios. A global likelihood function $\mathcal{L}$ is constructed from the latest ATLAS and CMS results. The Lilith inputs are the set of reduced couplings of the $125 \mathrm{GeV}$ state, i.e., couplings normalized by the SM ones, and possible invisible or undetected branching ratios. Note that Lilith can test Higgs bosons with masses within the $[123,128] \mathrm{GeV}$ interval, a warning will be issued if no such state can be 
found. In the case where two or more states have masses within this interval, their signal strengths will be summed incoherently and an effective Higgs state will be tested against the LHC measurements.

For a given parameter space point $\mathcal{P}$ of a new physics scenario, from which reduced couplings and new branching ratios can be obtained, Lilith returns ( -2 times) the loglikelihood evaluated at this point, $-2 \log \mathcal{L}(\mathcal{P})$, as,

$$
-2 \log \mathcal{L}(\mathcal{P}) \equiv \sum_{i=1}^{m}-2 \log \mathcal{L}_{i}^{\text {exp }}(\mathcal{P}),
$$

where $i$ indexes the $m$ measurements used to construct the global likelihood function $\mathcal{L}$ and $\mathcal{L}_{i}^{\text {exp }}$ are the individual experimental likelihood functions. If all the $\mathcal{L}_{i}^{\text {exp }}$ functions are Gaussian, $-2 \log \mathcal{L}(\mathcal{P})$ is identified as a chi-squared $\left(\chi^{2}\right)$. In practice, most of the measurements are well within the Gaussian regime, with the notable exception of the $H \rightarrow$ $Z Z^{*}$ measurements suffering from a small number of observed events. One could assess the statistical compatibility of $\mathcal{P}$ with the combined set of experimental results by computing a p-value based on a $\chi^{2}$ distribution with $n^{e x p} \equiv \sum_{i=1}^{m} n_{i}$ degrees of freedom, referred to as the number of experimental degrees of freedom, where $n_{i}$ is the number of variables of the likelihood function $\mathcal{L}_{i}^{\text {exp }}$. The number of experimental degrees of freedom is completely determined by the database of experimental results used within Lilith to construct the global Likelihood function $\mathcal{L}$. So far, experimental collaborations have delivered likelihood functions depending on $n_{i}=1,2$ variables (mostly identified as signal strengths), that can often be approximated by 1,2-dimensional Gaussian functions, respectively. ${ }^{1}$ This statistical interpretation is used by default within micrOMEGAs. We detail in the following the calculation of the p-value.

In general, the p-value is computed from a $\chi^{2}$ distribution with $n d f$ degrees of freedom, for an observed value of

$$
\Delta(-2 \log \mathcal{L}) \equiv-2 \log \mathcal{L}(\mathcal{P})-(-2 \log \mathcal{L}(\text { ref }))
$$

where $\mathcal{L}($ ref $)$ is a reference likelihood point. Explicitly, the p-value $p$ is obtained as

$$
p=\int_{\Delta(-2 \log \mathcal{L})}^{+\infty} \chi^{2}(x ; n d f) d x,
$$

where $\chi^{2}(x ; n d f)$ is the probability density function of a $\chi^{2}$ distribution with $n d f$ degrees of freedom and

$$
n d f \equiv n^{e x p}-n^{p a r},
$$

where $n^{e x p}$ are the experimental degrees of freedom and $n^{\text {par }}$ the number of relevant free parameters. $^{2}$ In micrOMEGAs, the two parameters $-2 \log \mathcal{L}($ ref) (a non-negative real) and $n^{\text {par }}$ (a non-negative integer) are free parameters and are dubbed m2logL_reference and n_par, respectively.

\footnotetext{
${ }^{1}$ Note that for 2-dimensional likelihood functions, the correlation between the two variables as determined by the experimental collaborations are completely accounted for.

${ }^{2}$ One should keep in mind, however, that in the case of a non-linear model, as obtained when parametrizing signal strengths by reduced couplings for instance, Eq. (4) may in fact be a very crude estimate of the real number of degrees of freedom; see Ref. [36] for a detailed discussion of this problem.
} 
By default in micrOMEGAs, both n_par and m2logL_reference are set to 0, corresponding to a p-value $p^{\text {def }}$ computed with $n^{e x p}$ degrees of freedom and $-2 \log \mathcal{L}($ ref $)=0$. The default $\mathrm{p}$-value is thus obtained as

$$
p^{\text {def }}=\int_{-2 \log \mathcal{L}(\mathcal{P})}^{+\infty} \chi^{2}\left(x ; n^{e x p}\right) d x .
$$

The file include/Lilith.inc (or Lilith.inc_f) contains the instructions to launch Lilith using a system call. The input file Lilith_in.xml for Lilith is created automatically via the command

\section{LilithMDL ("Lilith_in.xml")}

which returns the number of neutral Higgs particles and the file containing the list of reduced Higgs couplings as well as the branching ratios of Higgs decays to invisible, BR $_{\text {inv }}$, and to other non-SM final states, $\mathrm{BR}_{\text {undetected }}=1-\mathrm{BR}_{\mathrm{inv}}-\sum \mathrm{BR}(H \rightarrow \mathrm{SM} \mathrm{SM}){ }^{3}$. Note that the reduced couplings of the $125 \mathrm{GeV}$ Higgs are defined for all the models provided with micrOMEGAs with the exception of the $Z \gamma$ coupling. The latter is computed within Lilith assuming that only SM particles run in the loop. The command LilithMDL is model dependent but can be easily adapted for user-implemented models. In this case, the reduced couplings have to be defined in lib/lilith.c of the new model, including the effective Lagrangian for the loop-induced couplings. Alternatively micrOMEGAs contains a new option to generate automatically the input file. To use this option, it suffices to replace the above call to LilithMDL by

\section{LilithMO("Lilith_in.xml")}

The functionality of LilithMO is described in Section 2.3.

The input parameters n_par and m2logL_reference are defined in the main.c file of each model. The SLHA output file ${ }^{4}$, Lilith_out.slha, consists in six entries which are respectively $-2 \log \mathcal{L}(\mathcal{P}), n^{e x p},-2 \log \mathcal{L}($ ref $), n d f, p$ and the database version. For instance, for the mssmh . par point of the MSSM model, one obtains $-2 \log \mathcal{L}(\mathcal{P})=28.1285$ and $n^{e x p}=38$, leading to $p^{\text {def }}=0.879$. In this case the database DB_15.09 was used and the following SLHA output was generated,

$\begin{array}{cll}\text { BLOCK } & \text { LilithResults } & \\ 0 & 28.1285 & \#-2 * \text { LogL } \\ 1 & 38 & \# \text { exp_ndf } \\ 2 & 0.0 & \#-2 * \text { LogL_ref } \\ 3 & 38 & \# \text { fit_ndf } \\ 4 & 0.879 & \# \text { pvalue } \\ 5 & 15.09 & \# \text { database version }\end{array}$

The user is free to use the computed p-value at her/his will. For instance, one could flag or exclude points with too low p-value. In this context, a point with a p-value smaller than $0.3173,0.0455,0.0027$ could be excluded at more than the $1 \sigma, 2 \sigma, 3 \sigma$ levels, respectively.

\footnotetext{
${ }^{3} \mathrm{By}$ default, the automatic generation of the input file assumes that only DM contributes to the invisible width.

${ }^{4}$ We use here the name SLHA file to designate any file that has the BLOCK structure defined in SLHA $[37,38]$ even if the model is non supersymmetric.
} 


\begin{tabular}{|cc|cc|}
\hline $\begin{array}{c}-2 \log \mathcal{L}(\text { ref }) \\
(\text { m2logL_reference })\end{array}$ & $($ n_par $)$ & $\Delta(-2 \log \mathcal{L})$ & $n d f$ \\
\hline$-2 \log \mathcal{L}($ ref $)=0$ & $n^{\text {par }} \geq 0$ & $-2 \log \mathcal{L}(\mathcal{P})$ & $n^{\text {exp }}-n^{\text {par }}$ \\
\hline$-2 \log \mathcal{L}($ ref $)>0$ & $n^{\text {par }} \geq 1$ & $-2 \log \mathcal{L}(\mathcal{P})-(-2 \log \mathcal{L}($ ref $))$ & $n^{\text {par }}$ \\
\hline$-2 \log \mathcal{L}($ ref $)=-1$ & $n^{\text {par }} \geq 1$ & $-2 \log \mathcal{L}(\mathcal{P})-(-2 \log \mathcal{L}(\mathrm{SM}))$ & $n^{\text {par }}$ \\
\hline
\end{tabular}

Table 1: Roles of m2logL_reference and n_par in the Lilith p-value calculation within micrOMEGAs. The two left-most columns indicate the various possible inputs, while the two right-most columns give the corresponding parameters entering the p-value calculation of Eq. (3).

For generic values of m2logL_reference, Eq. (3) actually describes the p-value for a likelihood-ratio test in the asymptotic limit. Indeed, according to Wilk's theorem, such a statistical test is asymptotically distributed as a $\chi^{2}$ distribution, with $n d f \equiv n^{\text {par }} \neq 0$ standing for the number of free parameters controlling the Higgs sector of the scenario under study. Such a statistical procedure is well suited to perform a fit of the model, in which case one would take the best-fit point of the model, i.e., the point for which the global minimum of $-2 \log \mathcal{L}$ is attained, as the reference in Eq. (2). The best-fit point can be found by a preliminary scan or using a numerical minimization method for instance. Here, if m2logL_reference is set to -1 , the SM likelihood will be used as the reference point. As long as the SM is a good description of the $125 \mathrm{GeV}$ state properties, ${ }^{5}$ the SM likelihood should provide a good approximation of the best-fit point of any model with a SM limit. In the case of a large number of parameters, it may be justified to regard all reduced couplings as independent from each other, and thus identify $n^{\text {par }}$ as the number of reduced couplings that receive new physics contributions.

In Table 1, we summarize the roles of m2logL_reference and n_par, the two free parameters controlling the p-value calculation detailed in Eq. (3). By default, both n_par and $\mathrm{m} 2 \log L_{-} r e f e r e n c e$ are set to 0 , which corresponds to the first line of Table 1 with $n^{\text {par }}=0$. If only $\mathrm{n}_{\text {_par }}$ is given a non-vanishing value, the $\mathrm{p}$-value will be computed with a smaller number of degrees of freedom, as shown in the first line of the table. If m2logL_reference is set to a positive value, $\mathrm{n}_{-}$par should also be set to a value different from 0 (it is interpreted as the number of parameters controlling the Higgs sector of the given scenario) and the p-value will be computed with modified $n d f$ and $\Delta(-2 \log \mathcal{L})$ according to the second line of the table. On the other hand, if m2logL_reference is set to -1 , the SM will be used as the reference point in $\Delta(-2 \log \mathcal{L})$ (see Eq. (2)) as shown in the last line of Table 1.

\section{$2.2 \quad$ HiggsBounds and HiggsSignals}

Constraints on the properties of the $125 \mathrm{GeV}$ Higgs boson can also be obtained with HiggsSignals. Moreover exclusion limits provided by the experimental LHC and Teva-

\footnotetext{
${ }^{5}$ The SM provides an excellent fit to the current Higgs measurements; with Lilith DB_15.09, which contains all the Run 1 results, we find $-2 \log \mathcal{L}(\mathrm{SM})=25.9482$ with $n^{\exp }=38$, that is a $p$-value of $p^{\text {def }}=0.9312$.
} 
tron collaborations on additional Higgs bosons are obtained through an interface to HiggsBounds [24]. The interface to these codes has been updated with respect to previous versions, moreover theses codes are no longer distributed with micrOMEGAs but are downloaded when required. The file include/hBandS.inc contains the instructions to call both HiggsBounds and HiggsSignals, see [25] for more details on the input options.

In micrOMEGAs, the same SLHA file is used as an input to both of these codes, it relies on the effective coupling option for specifying the input. For models distributed with micrOMEGAs, the function

\section{hbBlocksMDL ("HB.in" , \&NchHiggs)}

can be used to write at the end of the SLHA file, HB. in, new blocks that contain the Higgs masses, the effective couplings of the Higgses normalized to the SM ones, including the reduced couplings squared to $\gamma \gamma, \gamma Z, g g$ as well as all Higgs total widths and branching ratios and the top decay width. A reference value is defined for the couplings that do not exist in the Standard Model. This routine returns the number of neutral Higgses, and NchHiggs gives the number of charged Higgs particles. For models not included in the micrOMEGAs distribution, the user can rewrite the function hbBlocksMDL located in the $1 \mathrm{ib}$ directory of the model or provide an SLHA file containing the reduced couplings squared. Moreover, the number of neutral and charged Higgs states must be provided, and the theoretical uncertainty on the masses of these particles must be specified in the BLOCK DMASS. Alternatively, the user can use the new option to generate automatically the input file by calling

$$
\text { hbBlocksMO ("HB. in" , \&NchHiggs) }
$$

The content of this function is described in Section 2.3. The complete outputs of HiggsBounds and HiggsSignals are stored in the files HB.out and HS.out respectively and can be accessed and read by the user using the slhaval function [39]. The screen output of micrOMEGAs contains the following information

$$
\text { HB(version number): result obsratio channel }
$$

where result $=0,1,-1$ denotes respectively whether a parameter point is excluded at 95\% CL, not excluded, or invalid; obsratio gives the ratio of the theoretical expectation relative to the observed value for the most constraining channel specified in channel. The HiggsSignals output displayed on the screen is simply

$$
\text { HS(version number): Nobservables } \operatorname{chi}^{`} 2 \text { pval }
$$

where Nobservables gives the number of observables used in the fit, chi ${ }^{\wedge} 2$ the associated $\chi^{2}$ and pval the p-value.

The interpretation of these values is left to the user. Note here that pval is determined in the same way as the default p-value in Lilith, see explanations to Eq. (5), unless the user explicitly provides a non-zero value for the number of parameters in the HiggsSignals input file HS. in, in which case $n d f=n o b s-n p a r$ is used. This gives a hypothesis test of how likely the model point explains the data, under the assumptions 
that all measurements (in all $\sim 100$ sub-categories considered in HiggsSignals) are nicely Gaussian; see however footnote 2 for caution about this definition of $n d f$.

To determine how much a model point is favoured or disfavoured over the SM, one can use $\Delta \chi^{2}$ to compute the likelihood ratio in the asymptotic limit as explained in the next-to-last paragraph of Section 2.1. We remind the reader that in this case the relevant number of degrees of freedom is the number of parameters (i.e. number of Higgs couplings receiving new physics contributions) instead of the number of observables. Identifying the $\mathrm{SM}$ as the best fit, the likelihood ratio simplifies to $p\left(\Delta \chi^{2} ; n p a r\right)$ with $\Delta \chi^{2}=\chi^{2}-\chi^{2}(\mathrm{SM})$.

\subsection{Automatic generation of interface files}

The functions LiLithMO and hbBlocksMO provided for generating automatically the input files for Lilith and HiggsBounds/HiggsSignals for new models contain two routines that allow to extract the couplings contained in the model file, lgrng1.mdl. These routines are described below. We stress however that they do not have to be called explicitly by the user. The first routine returns a description of a given vertex. The format used is

1Vert* Vv=getLagrVertex (name1, name2, name3, name4);

where name $1, . .$, name 4 are the names of the particles included in the vertex; for vertices with three particles, name4 should be replaced by NULL. The return parameter vv is the memory address of a structure which contains information about the vertex:

- $\mathrm{vv}->$ GGpower - power of strong coupling included in vertex

- $\mathrm{vv}->\mathrm{nTerms} \quad$ - number of different Lorentz structures in vertex

- $\mathrm{vv}->$ SymbVert [i] - text form of Lorentz structures $i \in[0, n$ Terms $]$

The second routine allows to obtain the numerical coefficients corresponding to each Lorentz structure. The command is

getNumCoeff (vv, coeff)

with coeff [i] the numerical coefficient for SymbVert [i]. Note that the strong coupling is factored out of the coefficients. For example, for the standard three gauge bosons interaction the SymbVert array and coefficients are

$$
\begin{aligned}
& \text { SymbVert }=\{\mathrm{p} 1 . \mathrm{m} 1 * \mathrm{~m} 3 . \mathrm{m} 2, \mathrm{p} 2 . \mathrm{m} 1 * \mathrm{~m} 3 . \mathrm{m} 2, \mathrm{p} 1 . \mathrm{m} 2 * \mathrm{~m} 3 . \mathrm{m} 1 \text {, } \\
& \mathrm{p} 2 . \mathrm{m} 2 * \mathrm{~m} 3 . \mathrm{m} 1, \mathrm{~m} 2 . \mathrm{m} 1 * \mathrm{p} 1 . \mathrm{m} 3, \mathrm{~m} 2 . \mathrm{m} 1 * \mathrm{p} 2 . \mathrm{m} 3\} \\
& \text { coeff }=\{x, 2 x,-2 x,-x, x, x\}
\end{aligned}
$$

where $x$ is the electromagnetic coupling $e$ for $W^{+} W^{-} \gamma$ and $x=\frac{e}{\tan \Theta_{W}}$ for $W^{+} W^{-} Z$. Using these two vertices, micrOMEGAs defines the electroweak parameters required for the computation of the reduced Higgs coupling in the model.

All the QCD-neutral scalars belonging to the even sector (not designated with ) are considered as Higgs particles. For each of these, micrOMEGAs calculates the couplings to SM fermions and massive bosons and writes down into the interface file the ratio of these couplings to the corresponding SM Higgs coupling. Note that the couplings of the Higgs 
to SM fermions can significantly depend on the QCD scale; micrOMEGAs assumes that the quark masses entering the vertices are obtained at the same scale in both the SM and the new model, thus the scale dependence in the reduced couplings to fermions disappears. The loop-induced couplings of the Higgs to gluons and photons are calculated by the LiLithMO/hbBlocksMO routines whether or not the $H g g$ and $H \gamma \gamma$ vertices are already implemented in the Lagrangian. This includes NLO-QCD corrections and is performed as described in $[11,40]$.

In addition to the reduced couplings, Lilith requires the branching ratios of the Higgs decays to invisibles and to other non-SM particles, while HiggsBounds/HiggsSignals requires all Higgs branching ratios as well as the total widths. These are also written automatically in the interface file (note however the caveat in footnote 3). When the Higgs widths and branching ratios are provided in an SLHA file, these values will be used. Otherwise LiLithMO/hbBlocksMO check the existence of $H \rightarrow g g$ and $H \rightarrow \gamma \gamma$ in the table of decays generated from the model Lagrangian. If found, the branching ratios and total widths are written in the interface file without comparing with the internal calculations. If not found, then LiLithMO/hbBlocksMO add these channels and recompute the total widths and all branching ratios.

\section{Collider limits on new particles}

\subsection{LEP limits}

Generic limits from LEP on charged supersymmetric particles have been implemented since the first version of micrOMEGAs [7]. The relevant function is

masslimits()

which returns a value greater than 1 and prints a Warning when the mass of at least one of the new particles conflicts with a direct limit on sparticle masses from LEP. The constraints on the Higgs sector from LEP are not implemented in this function.

The evaluation of two additional constraints are now provided. The first one is the limit on the invisible width of the $Z$ boson, $\Gamma_{i n v}(Z)<0.5 \mathrm{MeV}$ [41], which is relevant when the DM candidate is lighter than $M_{Z} / 2$. This can be checked by calling the function

Zinvisible()

which returns 1 and prints a Warning when $\Gamma_{i n v}(Z)>0.5 \mathrm{MeV}$. This function can be used in any model with one or two DM candidates where the $Z$ boson is defined by its PDG code (23). The second is the upper limit [42] on the cross section for the production of neutralinos $\sigma\left(e^{+} e^{-} \rightarrow \tilde{\chi}_{1}^{0} \tilde{\chi}_{i}^{0}\right), i \neq 1$, when the heavier neutralino decays into quark pairs and the LSP, $\tilde{\chi}_{i}^{0} \rightarrow \tilde{\chi}_{1}^{0} q \bar{q}$. The relevant function is

$$
\text { LspNIsp_LEP() }
$$

which returns $\sigma \times B R=\sum_{i} \sigma\left(e^{+} e^{-} \rightarrow \tilde{\chi}_{1}^{0} \tilde{\chi}_{i}^{0}\right) \times \mathrm{BR}\left(\tilde{\chi}_{i}^{0} \rightarrow \tilde{\chi}_{1}^{0} q \bar{q}\right)$ in pb as well as a flag greater than one if $\sigma \times B R>0.1(0.5) \mathrm{pb}$ if $m_{\mathrm{NLSP}}>(<) 100 \mathrm{GeV}$ [42]. This function can also be applied for non-SUSY models which feature the same signature, in this case the function will compute the cross section for production of the LSP and any other neutral particle from the odd sector which can decay into the LSP and a $Z$ boson. 


\subsection{LHC limits from SModelS}

The new particles present in extensions of the SM with a $\mathbb{Z}_{2}$ symmetry are strongly constrained by the LHC results. In particular, LHC limits on new (odd) particles can be obtained using SModelS [30-32], a code which tests Beyond the Standard Model (BSM) predictions against Simplified Model Spectra (SMS) results from searches for R-parity conserving SUSY by ATLAS and CMS. SModelS v1.1.0 decomposes any BSM model featuring a $\mathbb{Z}_{2}$ symmetry into its SMS components using a generic procedure where each SMS is defined by the vertex structure and the SM final state particles; BSM particles are described only by their masses, production cross sections and branching ratios. The underlying assumption is that differences in the event kinematics (e.g. from different production mechanisms or from the spin of the BSM particle) do not significantly affect the signal selection efficiencies. Within this assumption, SModels can be used for any BSM model with a $\mathbb{Z}_{2}$ symmetry as long as all heavier odd particles decay promptly to the dark matter candidate. ${ }^{6}$ Note that due to the $\mathbb{Z}_{2}$ symmetry only pair production is considered, and missing transverse energy (MET) is always implied in the final state description.

\subsubsection{Input files}

SModelS needs three files:

- an SLHA-type input file, containing the mass spectrum, decay tables ${ }^{7}$ and production cross sections for the parameter point under investigation;

- particles.py defining the particle content of the model, specifically which particles are even ("R-even") and which ones are odd ("R-odd") under the $\mathbb{Z}_{2}$ symmetry;

- a file for setting the run parameters, parameters.ini.

The first two are located in the same directory as main.c and are automatically written by micrOMEGAs by calling the function

smodels(Pcm, nf, csMinFb, fileName, wrt)

where Pcm is the proton beam energy in $\mathrm{GeV}$ and $\mathrm{nf}$ is the number of parton flavors used to compute the production cross sections of the $\mathbb{Z}_{2}$-odd particles. (Note that $u, d, \bar{u}$, $\bar{d}$ and gluons are always included while $s, c$, and $b$ quarks are included for $\mathrm{nf}=3,4,5$ respectively.) csMinFb defines the minimum production cross section in pb for $\mathbb{Z}_{2}$-odd particles; processes with lower cross sections are not added to the SLHA file passed to SModelS, here denoted by fileName. Finally, wrt is a steering flag for the screen output; if wrt $\neq 0$ the computed cross sections will be also written on the screen.

In the specific case of SUSY models, SModelS can be used to call nllFast [44] for the calculation of k-factors. The higher-order (NLO+NLL) cross sections for strong production processes are then added to the input file. For example the k-factors for $8 \mathrm{TeV}$ cross sections can be added via the system call

\footnotetext{
${ }^{6}$ Charged tracks may also be treated in an SMS context, see [43], and will be available in future versions of SModels.

${ }^{7}$ Note that all decay products in the decay table need to be on-shell.
} 
/runTools.py -particles ./ xseccomputer -p -s 8 -N -0 -f fileName

where fileName is the name of the SModels input file already used above. A file containting the instructions to call SModelS can be found in micromegas_4.3.4/include/SMODELS . inc (or SMODELS.inc_f) where fileName = smodels.in.

The SMS decomposition and confrontation against the LHC limits are also executed via a system call,

/runSModelS.py -f fileName -o ./ -p parameters.ini -particles ./ -v error

where -o sets the directory for the output file, -v controls the level of smodels output (only error messages will be printed in this example) and parameters.ini is the file that can be used to set the run parameters (see below). Running SModelS will produce two output files in the selected directory, a text summary in filename.smodels and an SLHA-type output in filename.smodelsslha. Note that a binary database is built when running SModelS for the first time. This can take a few minutes, but needs to be done only once.

Before testing against LHC limits, SModelS applies consistency checks on the input point, verifying e.g. that all decay tables are written in the input file and that all decays are kinematically allowed. It also checks whether there are long-lived charged particles and/or displaced vertices; in such a case the point is discarded and labelled as not tested. For all points passing the checks, SModelS proceeds to the decomposition into SMS components and matches them to the experimental upper limits in the database.

There are two types of results in the SModelS database. Upper limit (UL) maps directly report an upper limit on the topology weight, computed as production cross section times branching ratios, while efficiency maps (EM) report the signal selection efficiency. For EM type results SModelS collects all contributions to one signal region and calculates the cross section rescaled by the appropriate efficiencies. They can then be compared to an overall limit on the visible cross section. ${ }^{8}$

For each matching result, SModelS reports an $R$ value, defined as the ratio of the predicted theory cross section and the corresponding experimental upper limit. An $R$ value larger than 1 indicates that the point is excluded by the corresponding search. Additional information (expected $R$ value, $\chi^{2}$ and likelihood computation) are available for EM type results. In addition, SModelS returns information about important topologies for which no matching result exists. These so-called "missing topologies" are specified in the bracket notation defined in [30] and can be used to design new searches or Simplified Models that can constrain the scenario further.

Specific run parameters for SModelS can be set in a parameter file parameters.ini. A commented example can be found in Packages/smodels-v1.1.0patch1/parameters.ini. If no parameter file is specified, Packages/smodels-v1.1.0patch1/etc/parameters_default.ini is used. Concretely,

doInvisible and doCompress are used for turning on/off invisible and mass compression (on by default), turning on the former entails the compression of vertices where all SM particles are invisible in the detector (e.g., neutrinos).

\footnotetext{
${ }^{8}$ The current database version 1.1.0patch1 contains results from 25 ATLAS and 23 CMS SUSY searches at $8 \mathrm{TeV}$, and results from 3 early $13 \mathrm{TeV}$ searches. In addition, efficiency maps derived by the Fastlim collaboration [33] are also included in the database. The database can easily be updated with additional results independent of code updates [32].
} 
minmassgap (default $5 \mathrm{GeV}$ ) is the minimum mass gap for mass compression and

sigmacut (default $0.03 \mathrm{fb}$ ) is the cutoff cross section for topologies to be considered in the decomposition. Note that this value is independent from csMinFb that is only used by micrOMEGAs for writing the input file.

maxcond (default 0.2) sets the maximum condition violation for a result to be considered. Conditions are used when an experimental analysis combines final states with different selection efficiencies to evaluate a single upper limit (for example electron and muon final states). The condition is considered violated if the predicted composition differs from the one assumed in the limit setting procedure such that the constraint would be too strong. It is quantified as a relative difference from 0 (no violation) to 1 (condition maximally violated).

Additionally, the parameter . ini file allows to select only specific results from the database, by specifying analyses or txnames. For detailed explanations of these functionalities see [32].

\subsubsection{Output format}

An SLHA-type output format was designed for the SModelS-micrOMEGAs interface, and is written to filename.smodelsslha (in the directory selected by the user). This output consists of the blocks, SModelS_Settings, SModelS_Exclusion specifying the settings and constraints, and the blocks SModelS_Missing_Topos, SModelS_Outside_Grid, SModelS_Long_Cascade and SModelS_Asymmetric_Branches detailing information about the coverage by Simplified Models. Below we give a description of each block together with a sample output corresponding to the file mssm1.par in the MSSM directory.

- SModelS_Settings lists the SModelS code and database versions as well as input parameters for the decomposition. For example:

BLOCK SModelS_Settings
$\begin{array}{ll}0 & \mathrm{v} 1.1 .0 \text { patch } 1 \\ 1 & 1.1 .0 \text { patch } 1 \\ 2 & 0.2 \\ 3 & 1 \\ 4 & 5.0 \\ 5 & 0.03\end{array}$

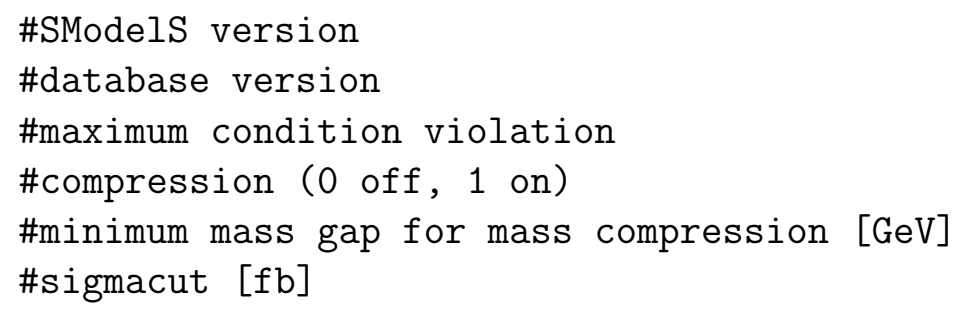

- SMode1S_Exclusion contains as the first line (the 00 entry) the status information if a point is excluded (1), not excluded (0), or not tested $(-1)$. The latter can occur in scenarios with long-lived charged particles or in scenarios where no matching SMS results are found.

If a point is excluded (status 1), this is followed by a list of all results with $R>1$, sorted by their $R$ values. For each of these results, the SMS topology identifier (entry 0) (so-called Tx-name, see [45] for an explanation of the terminology), the $R$ value (entry 1 ), for efficiency maps results the expected $R$ value (entry 2 ), a 
measure of condition violation (entry 3), and the analysis identifier (entry 4) are listed. Entries 5, 6 and 7 are relevant only for EM type results, and specify the most sensitive signal region (used for limit setting), the $\chi^{2}$ and the likelihood value respectively. If the point is not excluded (status 0 ), the result with the highest $R$ value is given instead to show whether a point is close to the exclusion limit or not.

In the example below, obtained from mssm1.par, the highest $R$ values correspond to a CMS supersymmetry search in the hadronic final states using $M_{T 2}$ [47] and a dijet+MET search constraining squark production, with $\tilde{q} \rightarrow q \tilde{\chi}_{1}^{0}$ obtained by ATLAS [46]. Note that only the first part of the file is reproduced.

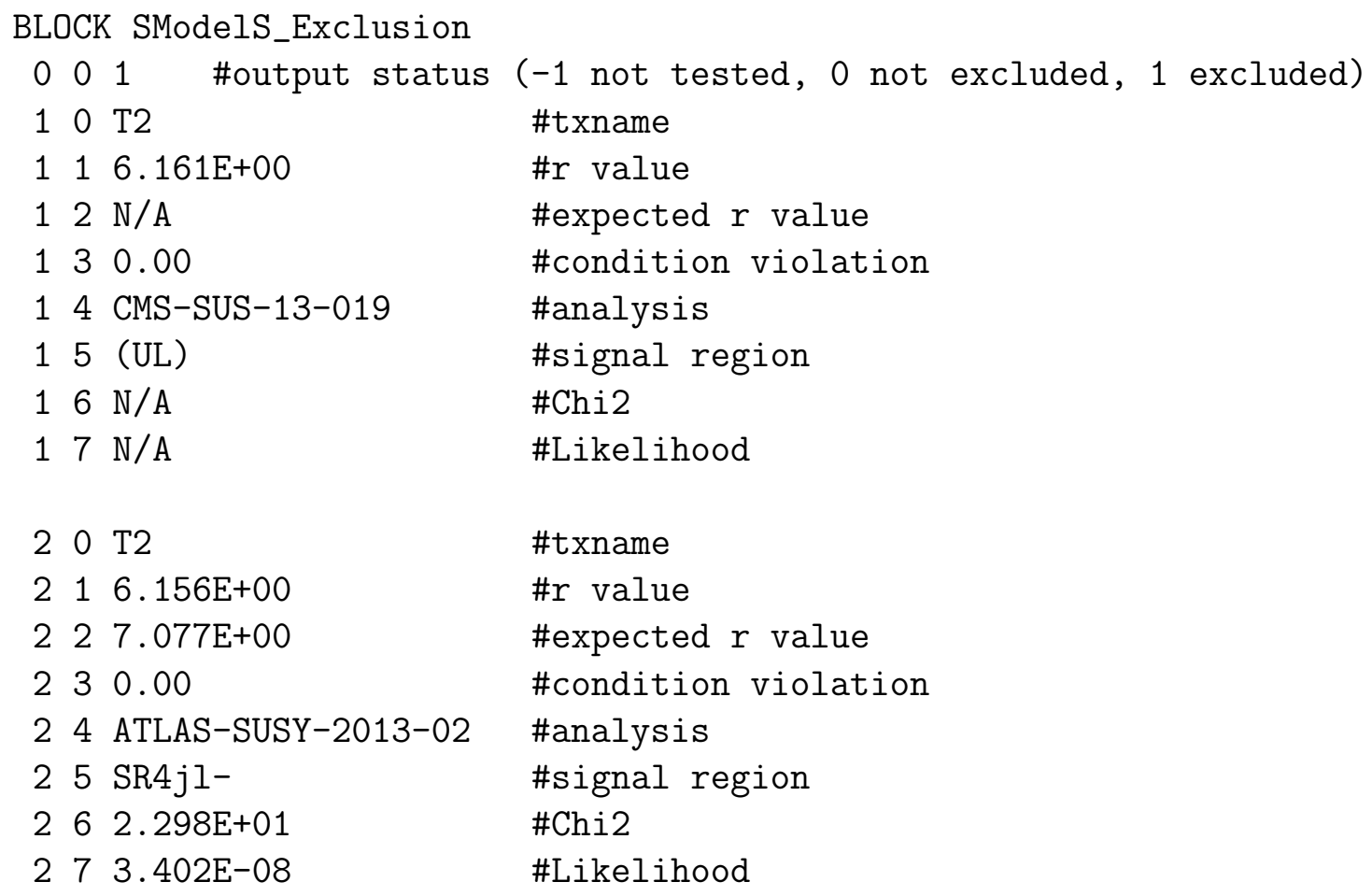

- SModelS_Missing_Topos lists up to ten missing topologies sorted by their weights $(=\sigma \times \mathrm{BR})$. Each entry consists of the line number, the $\sqrt{s}$ in $\mathrm{TeV}$, the weight and a description of the topology in the SModels bracket notation. Note that this information is useful mainly for points that are not excluded.

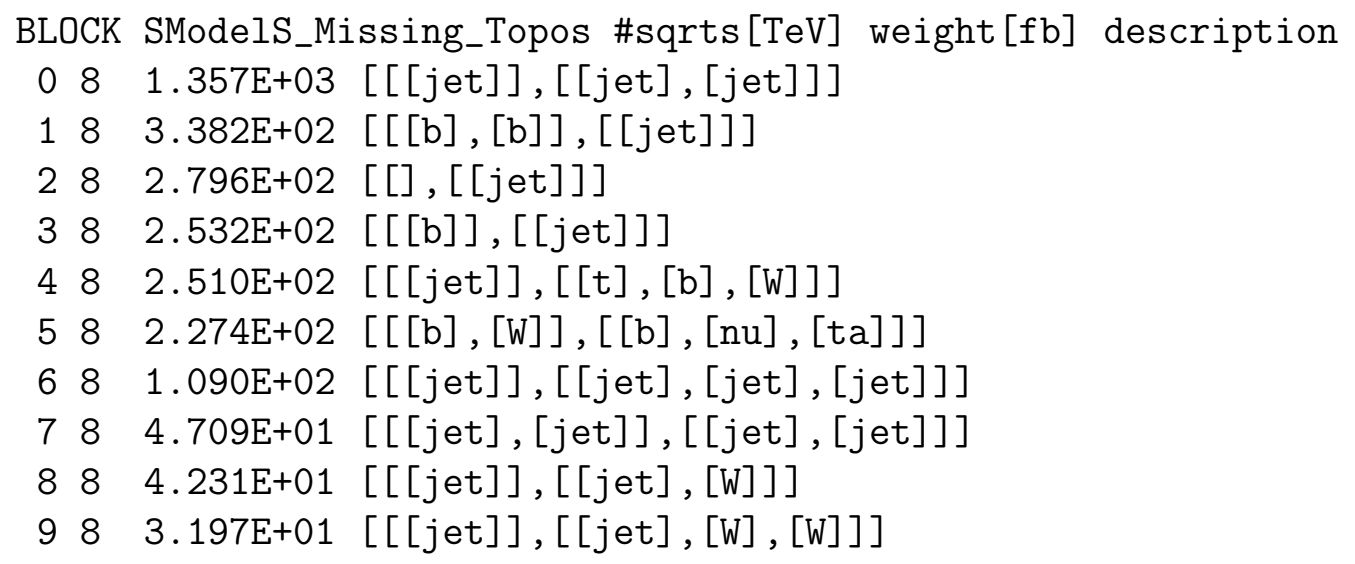


The blocks SModelS_Outside_Grid, SModelS_Long_Cascade and SModelS_Asymmetric_Branches are similar to the SMode1S_Missing_Topos block; we refer the reader to [32] for details.

\subsubsection{Identification of the SM-like Higgs}

Several SUSY searches exploit decay channels of heavy neutralinos to a SM-like Higgs and the LSP. For example, the three leptons ATLAS search [48] has an SMS interpretation for $\tilde{\chi}_{1}^{ \pm} \tilde{\chi}_{2}^{0}$ production, where $\tilde{\chi}_{1}^{ \pm} \rightarrow W \tilde{\chi}_{1}^{0}$ and $\tilde{\chi}_{2}^{0} \rightarrow h\left(\rightarrow W W^{*}\right.$ or $\left.Z Z^{*}\right) \tilde{\chi}_{1}^{0}$. To obtain generic results for a Higgs final state, the collaboration assumed SM branching ratios for the $h$, with the mass fixed to $m_{h}=125 \mathrm{GeV}$. To use these results it is important to identify the SM-like Higgs for any given parameter point. When calling smodels(), a check is performed on all neutral scalar particles with a mass in the range $123-128 \mathrm{GeV}$, comparing their branching ratios to $W W, Z Z, \tau \tau, b \bar{b}$ to those of a SM Higgs of the same mass. If they are compatible within 15\%, the corresponding particle will be identified as a SM Higgs by an entry of type

25 : "higgs",

-25 : "higgs"

in the rEven dictionary in the file particles.py. Note that the name higgs is reserved for a SM-like Higgs and should not be assigned generically. If no particle of that name is identified in particles.py, SModelS assumes that there is no SM-like Higgs, and the corresponding SMS results requiring a Higgs in the final state are not used to constrain the parameter point. However, for internal consistency, the name "higgs" has to be defined, and will be assigned to the PDG ID 12345.

When directly submitting an SLHA input file without calling smodels(), one has to make sure that a correct particles.py exists in the directory from which micrOMEGAs is run (the directory where main. $\mathrm{c}$ is located). For details on the syntax, see [31,32].

Note that the branching ratios of the SM Higgs are computed within micrOMEGAs, while the branching ratios in the new physics model are by default read from the SLHA input file. Differences in computing decays of the Higgs into off-shell particles or in the choice of fundamental constants can lead to the misidentification of the SM-like particle. If this occurs it is safer to simply disable the readout of the decay tables, such that all branching ratios will be consistently calculated by micrOMEGAs, see [11].

\subsubsection{SMS caveats}

A set of assumptions is introduced when defining the generic description of SMS. First, the production channel is not taken into account, and only on-shell particles are considered in the cascade decay. Virtual particles are replaced by an effective vertex, where only the on-shell decay products are specified. Additionally, new states are described only by their mass, neglecting all other quantum numbers, while in general different spin structures might modify selection efficiencies. Finally it should be noted that the SMS approach is only valid within the narrow width approximation. For a safe application of SModelS (in particular to non-MSSM scenarios), the above mentioned assumptions should be understood and if needed verified. The validity of these assumptions will depend on the concrete model under consideration, as well as details of the experimental search. In particular, an inclusive cut-and-count search might be less sensitive to differences than a shape-based analysis or a multivariate analysis. 
Previously, the effects of alternative production channels in squark simplified models were studied in [49], the effect of a different spin structure for the case of the dijet+missing transverse energy (MET) final state was studied in [50], and the effect of a different spin structure for the dilepton+MET final state was studied in [51]. Recently the spin dependence in $t \bar{t}+$ MET final states was tested in [52]. For all these cases it was found that the application of SMS limits is safe.

When the underlying assumptions that enter the simplified models interpretation are too restrictive to probe some parameter space of a model, more comprehensive recasting codes such as CheckMate [26,27] or MadAnalysis 5 [28,29], should be used instead. These codes however require generating events for the new physics signal and are therefore more computer-time consuming and less adapted to large scans. Recasting by event simulation is not directly interfaced in this distribution.

\subsection{Other simplified-model limits}

\subsection{1 $\quad Z^{\prime}$ searches}

Limits on a new massive Abelian gauge boson from various searches at the LHC are taken into account in micrOMEGAs through a routine originally designed for the UMSSM model $[53,54]$ but which can be adapted to other models with a $Z^{\prime}$ uniquely defined by the PDG code 32 . The usage is

\section{Zprimelimits()}

which returns 0 if the point in the parameter space of the model is not excluded by the $Z^{\prime}$ constraints, 1 if the point is excluded and 2 if both subroutines dealing with $Z^{\prime}$ constraints cannot test the given scenario.

Currently two types of searches defined in different subroutines of Zprimelimits () are implemented. The latest $Z^{\prime}$ search in the dilepton final state at $\sqrt{s}=13 \mathrm{TeV}$ from ATLAS [55] is considered in the first subroutine Zprime_dilepton. It excludes, for instance, a Sequential Standard Model (SSM) $Z^{\prime}$ up to $3.36 \mathrm{TeV}$. The evaluation done by ATLAS however assumes that the $Z^{\prime}$ only decays into SM particles. The limit can therefore be relaxed if the $Z^{\prime}$ also has decay modes into new particles, thus reducing the branching ratio into dileptons. For this reason, after the cross section $\sigma\left(p p \rightarrow Z^{\prime}\right)$ at $\sqrt{s}=13 \mathrm{TeV}$ is computed with the hCollider function of micrOMEGAs, a rescaling factor is applied to $\sigma\left(p p \rightarrow Z^{\prime}\right) \times \operatorname{BR}\left(Z^{\prime} \rightarrow l^{+} l^{-}\right)$, where $l^{+} l^{-}$stands for the combined dielectron and dimuon channels, to match the computed cross section in the limit where $Z^{\prime}$ only decays into SM particles used by ATLAS. This is then compared with the limit set by ATLAS in the range $M_{Z^{\prime}} \in[0.5 ; 4] \mathrm{TeV}$. The subroutine returns 1 if $\sigma\left(p p \rightarrow Z^{\prime}\right) \times \mathrm{BR}\left(Z^{\prime} \rightarrow l^{+} l^{-}\right)$ exceeds the observed limit.

If the scenario considered is allowed or not tested by Zprime_dilepton, a second subroutine called Zprime_dijet analyses the point using constraints from LHC dijet searches at $\sqrt{s}=8 \mathrm{TeV}[56-58]$ and at $\sqrt{s}=13 \mathrm{TeV}[59,60]$. This subroutine uses the recasting performed in [61] for a combination of ATLAS and CMS searches. The recasting provides an upper bound at $95 \% \mathrm{CL}$ on $g_{q}^{2} \times \mathrm{BR}\left(Z^{\prime} \rightarrow j j\right)$ where $g_{q}$ is the coupling of $Z^{\prime}$ to quarks $u, d, c, s$ or $b$ for set of values for $\left\{M_{Z^{\prime}}, \Gamma / M_{Z^{\prime}}\right\}$ where $\Gamma$ is the total $Z^{\prime}$ width. Only quarks (except top) and invisible particles are included in the calculation of the $Z^{\prime}$ width. For the relevant $\left\{M_{Z^{\prime}}, \Gamma / M_{Z^{\prime}}\right\}$, Zprime_dijet compares this upper bound with 
the value of $\sum_{q}\left[\left(g_{q}^{V}\right)^{2}+\left(g_{q}^{A}\right)^{2}\right] \times \mathrm{BR}\left(Z^{\prime} \rightarrow q \bar{q}\right)$ corresponding to the scenario considered : it returns 1 if the result exceeds the upper bound and 0 otherwise. Here $q=u, d, c, s, b$; $g_{q}^{V}$ and $g_{q}^{V}$ are the vectorial and axial couplings of $Z^{\prime}$ to $q$ which have to be defined in the model files. If $M_{Z^{\prime}}>4 \mathrm{TeV}$ or no coupling is defined this subroutine cannot be used and returns automatically 2. Note finally that Zprimelimits() returns 1 if $M_{Z^{\prime}}<0.5 \mathrm{TeV}$ and 2 for points for which the narrow-width approximation is not valid, i.e. $\Gamma / M_{Z^{\prime}}>0.3$.

\subsubsection{Mono-jet searches}

At the LHC, DM searches mainly proceed via mono- $X$ signatures, where $X$ indicates any visible, i.e. collider detectable, particle produced in association with a DM pair. These signatures are characterised by a high- $p_{T}$ object recoiling against MET. Currently, the most stringent limits arise from the $8 \mathrm{TeV}$ mono-jet searches [62,63], while early $13 \mathrm{TeV}$ results are quickly reaching the Run-1 sensitivity [64]. The function

monoJet (pName1, pName2)

computes the cross section for $p, p \rightarrow$ pName1, pName $2+j e t$ at $\sqrt{s}=8 \mathrm{TeV}$ where pName1, pName 2 are the names of neutral outgoing particles and jet includes light quarks $(\mathrm{u}, \mathrm{d}, \mathrm{s})$ and gluons. The cross section is computed through a built-in map that takes into account the differences between parton level and detector reconstructed events as well as next to leading order corrections to the signal prediction. ${ }^{9}$ This map has been created via the MadGraph5_aMC@NLO [66], PYTHIA8 [67] and CheckMate [26] chain for various MET selections and mediator masses. The function convolutes the parton level prediction with this map to compute the final rates for each signal region (SR) of the CMS analysis [62], which are then compared with the experimental background and data. The function returns the resulting CL obtained with the CLs technique $[68,69]$ for each SR and chooses the most constraining one. We checked that in the case of a vector mediator, the exclusion levels match those provided by CMS.

\section{Installation and sample output}

The code can be downloaded from [70]. After unpacking, go to the micromegas_4.3.4 directory and type

make or gmake

To work with one of the models already included in the distribution, go to the relevant model directory (for example MSSM)

cd MSSM

The file main.c is a sample program to illustrate the usage of the functionalities described in this paper. It contains at the top of the code various options to steer the behaviour of

\footnotetext{
${ }^{9}$ We have used the NLO DM model publicly available at the FeynRules [65] page http://feynrules . irmp.ucl.ac.be/wiki/DMsimp.
} 
the code. For example

\#define RGE suspect

\#define EWSB

\#define MASSES_INFO

\#define CONSTRAINTS

\#define HIGGSBOUNDS

\#define HIGGSSIGNALS

\#define LILITH

\#define SMODELS

will compute the MSSM spectrum using SuSpect with input parameters specified at the electroweak scale (to be provided in an input file, for example mssms.par, as described in [7]). The resulting mass spectrum will be displayed on the screen together with various LEP and flavour constraints, including the ones discussed in Section 3.1. In this example, the Higgs constraints will be checked by HiggsBounds and HiggsSignals as well as with Lilith. Note, however, that the latter two perform the same task and only one should be used in, e.g., a global fit. The LHC constraints on the SUSY spectrum will be checked with SModels. ${ }^{10}$

The codes SuSpect, Lilith and SModelS are distributed with micrOMEGAs and are located in the micromegas_4.3.4/Packages directory. The codes HiggsBounds and HiggsSignals, when requested, will be copied automatically from a repository upon compilation of micrOMEGAs. They will then also be stored in the micromegas_4.3.4/Packages directory.

Compiling and running main.c with the above options and the input file mssms.par

make main=main.c

./main mssms.par

will produce the following output

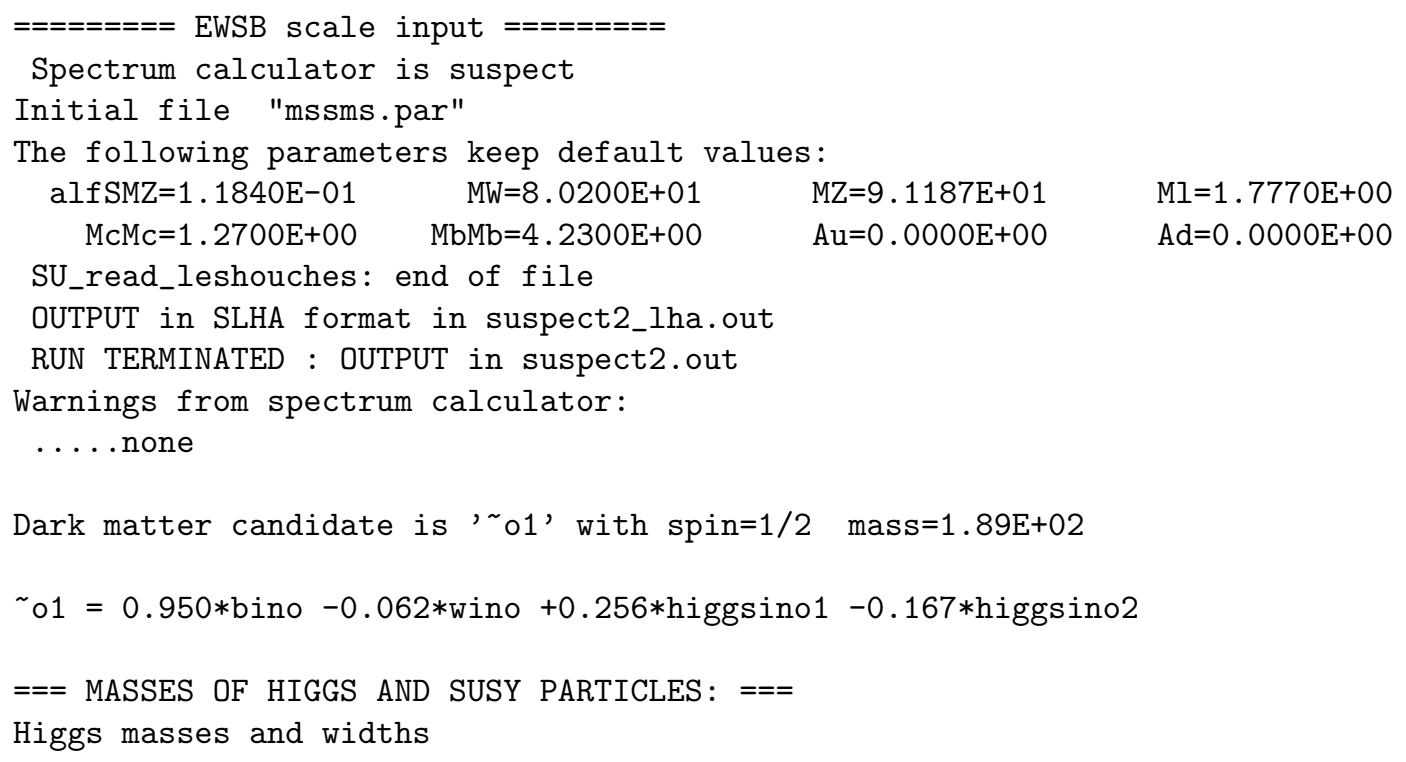

\footnotetext{
${ }^{10}$ Running SModelS requires computing production cross sections for all non standard particles in the model. The compilation of these processes at the first call of micrOMEGAs may take a few minutes.
} 


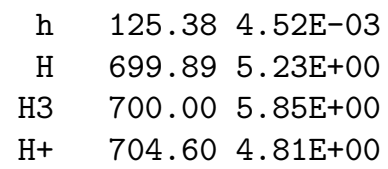

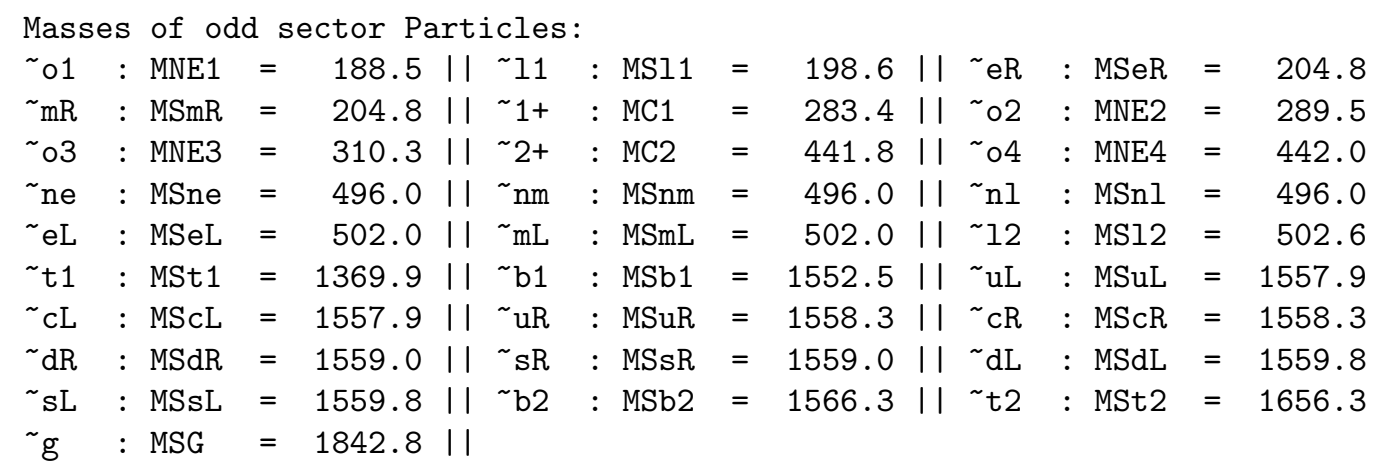

$====$ Physical Constraints: $=====$

deltartho=3.44E-04

gmuon=1.12E-09

bsgnlo $=3.21 \mathrm{E}-04(\mathrm{SM} 3.28 \mathrm{E}-04)$

bsmumu=3.28E-09

btaunu=9.59E-01

dtaunu $=5.17 \mathrm{E}-02$ dmunu=5.33E-03

$\mathrm{R} 123=9.998 \mathrm{E}-01$

MassLimits OK

$\mathrm{HB}(4.3 .1)$ : result=1 obsratio=5.15E-01 channel= (pp) $->\mathrm{h} 2->$ tautau, using $-2 \ln (\mathrm{L})$ reconstruction (CMS-HIG-PAS 14-029)

HS (1.4.0): Nobservables $=89 \mathrm{chi}^{\uparrow} 2=7.74 \mathrm{E}+01 \mathrm{pval}=8.06 \mathrm{E}-01$

LILITH (DB15.09): $\quad-2 * \log (\mathrm{L}): 28.14 ;-2 * \log ($ L_reference) : $0.00 ;$ ndf: 38; p-value: $8.79 \mathrm{E}-01$

SMODELS :

found SM-like Higgs $=\mathrm{h}$

v1.1.0patch1 with database 1.1.0patch1

highest $\mathrm{R}=3.16 \mathrm{E}-01$ from ATLAS-CONF-2013-047, topology T2

not excluded.

The user can also implement his/her own model in micrOMEGAs, this requires creating model files in the CalcHEP format, see [8] for details. The command

\section{./newProject ModelName}

will create a directory ModelName containing sample main files that include the functions necessary to compute all dark matter observables and collider constraints.

\section{Conclusions}

Version 4.3.1 of micrOMEGAs allows for a fast an efficient exploitation of the collider results on new particle searches and on Higgs properties. This is achieved through interfaces with Lilith [22] and HiggsSignals [23] for checking the Higgs signal strengths, HiggsBounds [24,25] to check limits on additional neutral and charged Higgs states, and SModelS [30-32] to check the LHC limits on the production of new odd particles in the 
context of Simplified Model Spectra constraints. In addition, in-house routines are provided for checking LEP constraints, limits on a new $Z^{\prime}$ at the LHC, and LHC limits from mono-jet searches. For each of these codes, we explained the basic functionality, the usage within micrOMEGAs the input format and the interpretation of the output.

micrOMEGAs thus provides a general framework for evaluating collider constraints on dark matter models. The framework is fast enough to be suitable for scans of parameter space. It is also quite generic and thus applicable to a wide range of models, including new ones that can be added to micrOMEGAs. Of course some limitations apply. For example, the Higgs signal strength approach in Lilith and HiggsSignals applies only to Higgs sectors with the same tensor structure and Higgs production modes as in the SM. Moreover, the SMS approach used in SModelS is subject to a few caveats explained in the paper. Finally, the $Z^{\prime}$ limits are applicable only to models with one extra $Z$ boson. The user is responsible for observing these limitations.

\section{Acknowledgements}

We thank Tim Stefaniak for discussions on HiggsSignals. This work was supported in part by the LIA-TCAP of CNRS, by the French ANR, Project DMAstro-LHC ANR-12BS05-0006, the Theory-LHC-France Initiative of INP/IN2P3, the Investissements d'avenir, Labex ENIGMASS, and by the Research Executive Agency (REA) of the European Union under the Grant Agreement PITN-GA2012-316704 ("HiggsTools"). The work of AP was also supported by the Russian foundation for Basic Research, grant RFBR-15-52-16021CNRS-a.

\section{References}

[1] G. Bertone, D. Hooper, and J. Silk, "Particle dark matter: Evidence, candidates and constraints," Phys. Rept. 405 (2005) 279-390, arXiv:hep-ph/0404175 [hep-ph] .

[2] J. Silk et al., "Particle Dark Matter: Observations, Models and Searches," Cambridge University Press (2010).

[3] G. Jungman, M. Kamionkowski, and K. Griest, "Supersymmetric dark matter," Phys. Rept. 267 (1996) 195-373, arXiv:hep-ph/9506380 [hep-ph].

[4] K. Griest and M. Kamionkowski, "Supersymmetric dark matter," Phys. Rept. 333 (2000) 167-182.

[5] J. McDonald, "Gauge singlet scalars as cold dark matter," Phys. Rev. D50 (1994) 3637-3649, arXiv:hep-ph/0702143 [HEP-PH] .

[6] B. Patt and F. Wilczek, "Higgs-field portal into hidden sectors," arXiv:hep-ph/0605188 [hep-ph].

[7] G. Belanger, F. Boudjema, A. Pukhov, and A. Semenov, "MicrOMEGAs: A Program for calculating the relic density in the MSSM," Comput. Phys. Commun. 149 (2002) 103-120, arXiv:hep-ph/0112278 [hep-ph]. 
[8] G. Belanger, F. Boudjema, A. Pukhov, and A. Semenov, "MicrOMEGAs 2.0: A Program to calculate the relic density of dark matter in a generic model," Comput. Phys. Commun. 176 (2007) 367-382, arXiv:hep-ph/0607059 [hep-ph].

[9] G. Belanger, F. Boudjema, A. Pukhov, and A. Semenov, "Dark matter direct detection rate in a generic model with micrOMEGAs 2.2," Comput. Phys. Commun. 180 (2009) 747-767, arXiv:0803.2360 [hep-ph].

[10] G. Belanger, F. Boudjema, P. Brun, A. Pukhov, S. Rosier-Lees, P. Salati, and A. Semenov, "Indirect search for dark matter with micrOMEGAs2.4," Comput. Phys. Commun. 182 (2011) 842-856, arXiv:1004.1092 [hep-ph].

[11] G. Belanger, F. Boudjema, A. Pukhov, and A. Semenov, "micrOMEGAs_3: A program for calculating dark matter observables," Comput. Phys. Commun. 185 (2014) 960-985, arXiv:1305.0237 [hep-ph].

[12] G. Belanger, F. Boudjema, A. Pukhov, and A. Semenov, "micrOMEGAs4.1: two dark matter candidates," Comput. Phys. Commun. 192 (2015) 322-329, arXiv:1407.6129 [hep-ph].

[13] A. Pukhov, "CalcHEP 2.3: MSSM, structure functions, event generation, batchs, and generation of matrix elements for other packages," arXiv:hep-ph/0412191 [hep-ph].

[14] A. Belyaev, N. D. Christensen, and A. Pukhov, "CalcHEP 3.4 for collider physics within and beyond the Standard Model," Comput. Phys. Commun. 184 (2013) 1729-1769, arXiv:1207.6082 [hep-ph].

[15] ATLAS Collaboration, G. Aad et al., "Observation of a new particle in the search for the Standard Model Higgs boson with the ATLAS detector at the LHC," Phys. Lett. B716 (2012) 1-29, arXiv:1207.7214 [hep-ex].

[16] CMS Collaboration, S. Chatrchyan et al., "Observation of a new boson at a mass of $125 \mathrm{GeV}$ with the CMS experiment at the LHC," Phys. Lett. B716 (2012) 30-61, arXiv:1207.7235 [hep-ex].

[17] https:

//twiki.cern.ch/twiki/bin/view/AtlasPublic/SupersymmetryPublicResults.

[18] https://twiki.cern.ch/twiki/bin/view/AtlasPublic/ExoticsPublicResults.

[19] https://twiki.cern.ch/twiki/bin/view/CMSPublic/PhysicsResultsSUS.

[20] https://twiki.cern.ch/twiki/bin/view/CMSPublic/PhysicsResultsEXO.

[21] https://twiki.cern.ch/twiki/bin/view/CMSPublic/PhysicsResultsB2G.

[22] J. Bernon and B. Dumont, "Lilith: a tool for constraining new physics from Higgs measurements," Eur. Phys. J. C75 no. 9, (2015) 440, arXiv:1502.04138 [hep-ph] . 
[23] P. Bechtle, S. Heinemeyer, O. Stål, T. Stefaniak, and G. Weiglein, "HiggsSignals: Confronting arbitrary Higgs sectors with measurements at the Tevatron and the LHC," Eur. Phys. J. C74 (2014) 2711, arXiv:1305.1933 [hep-ph].

[24] P. Bechtle, O. Brein, S. Heinemeyer, G. Weiglein, and K. E. Williams, "HiggsBounds 2.0.0: Confronting Neutral and Charged Higgs Sector Predictions with Exclusion Bounds from LEP and the Tevatron," Comput. Phys. Commun. 182 (2011) 2605-2631, arXiv:1102.1898 [hep-ph].

[25] P. Bechtle, O. Brein, S. Heinemeyer, O. Stål, T. Stefaniak, et al., "HiggsBounds - 4: Improved Tests of Extended Higgs Sectors against Exclusion Bounds from LEP, the Tevatron and the LHC," Eur. Phys. J. C74 (2014) 2693, arXiv:1311.0055 [hep-ph] .

[26] M. Drees, H. Dreiner, D. Schmeier, J. Tattersall, and J. S. Kim, "CheckMATE: Confronting your Favourite New Physics Model with LHC Data," Comput. Phys. Commun. 187 (2015) 227-265, arXiv:1312.2591 [hep-ph].

[27] J. S. Kim, D. Schmeier, J. Tattersall, and K. Rolbiecki, "A framework to create customised LHC analyses within CheckMATE," Comput. Phys. Commun. 196 (2015) 535-562, arXiv:1503.01123 [hep-ph].

[28] B. Dumont, B. Fuks, S. Kraml, S. Bein, G. Chalons, E. Conte, S. Kulkarni, D. Sengupta, and C. Wymant, "Toward a public analysis database for LHC new physics searches using MADANALYSIS 5," Eur. Phys. J. C75 no. 2, (2015) 56, arXiv:1407.3278 [hep-ph].

[29] E. Conte, B. Dumont, B. Fuks, and C. Wymant, "Designing and recasting LHC analyses with MadAnalysis 5," Eur. Phys. J. C74 no. 10, (2014) 3103, arXiv:1405.3982 [hep-ph].

[30] S. Kraml, S. Kulkarni, U. Laa, A. Lessa, W. Magerl, D. Proschofsky-Spindler, and W. Waltenberger, "SModelS: a tool for interpreting simplified-model results from the LHC and its application to supersymmetry," Eur. Phys. J. C74 (2014) 2868, arXiv:1312.4175 [hep-ph].

[31] S. Kraml, S. Kulkarni, U. Laa, A. Lessa, V. Magerl, W. Magerl, D. Proschofsky-Spindler, M. Traub, and W. Waltenberger, "SModelS v1.0: a short user guide," arXiv:1412.1745 [hep-ph].

[32] F. Ambrogi et al., "SModelS v1.1 user manual," arXiv:1701.06586 [hep-ph].

[33] M. Papucci, K. Sakurai, A. Weiler, and L. Zeune, "Fastlim: a fast LHC limit calculator," Eur. Phys. J. C74 no. 11, (2014) 3163, arXiv:1402.0492 [hep-ph].

[34] D. Barducci, A. Belyaev, M. Buchkremer, J. Marrouche, S. Moretti, and L. Panizzi, "XQCAT: eXtra Quark Combined Analysis Tool," Comput. Phys. Commun. 197 (2015) 263-275, arXiv:1409.3116 [hep-ph] .

[35] S. Caron, J. S. Kim, K. Rolbiecki, R. Ruiz de Austri, and B. Stienen, "The BSM-AI project: SUSY-AI - Generalizing LHC limits on Supersymmetry with Machine Learning," arXiv:1605.02797 [hep-ph]. 
[36] R. Andrae, T. Schulze-Hartung, and P. Melchior, "Dos and don'ts of reduced chi-squared," arXiv:1012.3754 [astro-ph.IM].

[37] P. Z. Skands, B. Allanach, H. Baer, C. Balazs, G. Belanger, et al., "SUSY Les Houches accord: Interfacing SUSY spectrum calculators, decay packages, and event generators," JHEP 0407 (2004) 036, arXiv:hep-ph/0311123 [hep-ph] .

[38] B. C. Allanach et al., "SUSY Les Houches Accord 2," Comput. Phys. Commun. 180 (2009) 8-25, arXiv:0801.0045 [hep-ph].

[39] G. Belanger, F. Boudjema, and A. Pukhov, "micrOMEGAs : a code for the calculation of Dark Matter properties in generic models of particle interaction," in The Dark Secrets of the Terascale, pp. 739-790. 2013. arXiv:1402.0787 [hep-ph].

[40] A. Djouadi, J. Kalinowski and M. Spira, "HDECAY: A Program for Higgs boson decays in the standard model and its supersymmetric extension," Comput. Phys. Commun. 108 (1998) 56, [hep-ph/9704448].

[41] A. Freitas, "Higher-order electroweak corrections to the partial widths and branching ratios of the Z boson," JHEP 04 (2014) 070, arXiv: 1401.2447 [hep-ph].

[42] OPAL Collaboration, G. Abbiendi et al., "Search for chargino and neutralino production at $\sqrt{s}=192-209 \mathrm{GeV}$ at LEP," Eur. Phys. J. C35 (2004) 1-20, arXiv:hep-ex/0401026 [hep-ex].

[43] J. Heisig, A. Lessa, and L. Quertenmont, "Simplified Models for Exotic BSM Searches," arXiv:1509.00473 [hep-ph].

[44] http:

//pauli.uni-muenster.de/\$`sim\$akule_01/nllwiki/index.php/NLL-fast.

[45] http://smodels.hephy.at/wiki/SmsDictionary

[46] ATLAS Collaboration, G. Aad et al., "Search for squarks and gluinos with the ATLAS detector in final states with jets and missing transverse momentum using $\sqrt{s}=8 \mathrm{TeV}$ proton-proton collision data," JHEP 09 (2014) 176, arXiv: 1405.7875 [hep-ex].

[47] CMS Collaboration, V. Khachatryan et al., "Searches for Supersymmetry using the $\mathrm{M}_{T 2}$ Variable in Hadronic Events Produced in pp Collisions at $8 \mathrm{TeV}$," JHEP 05 (2015) 078, arXiv:1502.04358 [hep-ex].

[48] ATLAS Collaboration, G. Aad et al., "Search for direct production of charginos and neutralinos in events with three leptons and missing transverse momentum in $\sqrt{s}=8 \mathrm{TeV} p p$ collisions with the ATLAS detector," JHEP 04 (2014) 169, arXiv:1402.7029 [hep-ex].

[49] L. Edelhäuser, J. Heisig, M. Krämer, L. Oymanns, and J. Sonneveld, "Constraining supersymmetry at the LHC with simplified models for squark production," JHEP 12 (2014) 022, arXiv:1410.0965 [hep-ph]. 
[50] L. Edelhäuser, M. Krämer, and J. Sonneveld, "Simplified models for same-spin new physics scenarios," JHEP 04 (2015) 146, arXiv:1501.03942 [hep-ph].

[51] C. Arina, M. E. C. Catalan, S. Kraml, S. Kulkarni, and U. Laa, "Constraints on sneutrino dark matter from LHC Run 1," JHEP 05 (2015) 142, arXiv:1503.02960 [hep-ph] .

[52] S. Kraml, U. Laa, L. Panizzi and H. Prager, "Scalar versus fermionic top partner interpretations of $t \bar{t}+E_{T}^{\text {miss }}$ searches at the LHC," JHEP 11 (2016) 107, arXiv: 1607.02050 [hep-ph].

[53] G. Belanger, J. Da Silva, and A. Pukhov, "The Right-handed sneutrino as thermal dark matter in U(1) extensions of the MSSM," JCAP 1112 (2011) 014, arXiv:1110.2414 [hep-ph].

[54] G. Belanger, J. Da Silva, U. Laa, and A. Pukhov, "Probing U(1) extensions of the MSSM at the LHC Run I and in dark matter searches," JHEP 09 (2015) 151, arXiv:1505.06243 [hep-ph].

[55] ATLAS Collaboration, M. Aaboud et al., "Search for high-mass new phenomena in the dilepton final state using proton-proton collisions at $\sqrt{s}=13 \mathrm{TeV}$ with the ATLAS detector," arXiv:1607.03669 [hep-ex].

[56] ATLAS Collaboration, G. Aad et al., "Search for new phenomena in the dijet mass distribution using $p-p$ collision data at $\sqrt{s}=8 \mathrm{TeV}$ with the ATLAS detector," Phys. Rev. D91 no. 5, (2015) 052007, arXiv:1407.1376 [hep-ex].

[57] CMS Collaboration, V. Khachatryan et al., "Search for resonances and quantum black holes using dijet mass spectra in proton-proton collisions at $\sqrt{s}=8 \mathrm{TeV}$," Phys. Rev. D91 no. 5, (2015) 052009, arXiv:1501.04198 [hep-ex].

[58] CMS Collaboration, V. Khachatryan et al., "Search for narrow resonances in dijet final states at $\sqrt{(s)}=8 \mathrm{TeV}$ with the novel CMS technique of data scouting," Phys. Rev. Lett. 117 no. 3, (2016) 031802, arXiv:1604.08907 [hep-ex].

[59] ATLAS Collaboration, G. Aad et al., "Search for new phenomena in dijet mass and angular distributions from $p p$ collisions at $\sqrt{s}=13 \mathrm{TeV}$ with the ATLAS detector," Phys. Lett. B754 (2016) 302-322, arXiv:1512.01530 [hep-ex].

[60] CMS Collaboration, V. Khachatryan et al., "Search for narrow resonances decaying to dijets in proton-proton collisions at $\sqrt{(s)}=13 \mathrm{TeV}$," Phys. Rev. Lett. 116 no. 7, (2016) 071801, arXiv:1512.01224 [hep-ex].

[61] M. Fairbairn, J. Heal, F. Kahlhoefer, and P. Tunney, "Constraints on Z' models from LHC dijet searches," arXiv:1605.07940 [hep-ph].

[62] CMS Collaboration, V. Khachatryan et al., "Search for dark matter, extra dimensions, and unparticles in monojet events in proton-proton collisions at $\sqrt{s}=8$ TeV," Eur. Phys. J. C75 no. 5, (2015) 235, arXiv:1408.3583 [hep-ex]. 
[63] ATLAS Collaboration, G. Aad et al., "Search for new phenomena in final states with an energetic jet and large missing transverse momentum in pp collisions at $\sqrt{s}=8 \mathrm{TeV}$ with the ATLAS detector," Eur. Phys. J. C75 no. 7, (2015) 299, arXiv:1502.01518 [hep-ex]. [Erratum: Eur. Phys. J.C75,no.9,408(2015)].

[64] CMS Collaboration, "Search for dark matter with jets and missing transverse energy at 13 TeV," CMS-PAS-EXO-15-003.

[65] A. Alloul, N. D. Christensen, C. Degrande, C. Duhr, and B. Fuks, "FeynRules 2.0 A complete toolbox for tree-level phenomenology," Comput. Phys. Commun. 185 (2014) 2250-2300, arXiv:1310.1921 [hep-ph].

[66] J. Alwall, R. Frederix, S. Frixione, V. Hirschi, F. Maltoni, O. Mattelaer, H. S. Shao, T. Stelzer, P. Torrielli, and M. Zaro, "The automated computation of tree-level and next-to-leading order differential cross sections, and their matching to parton shower simulations," JHEP 07 (2014) 079, arXiv:1405.0301 [hep-ph].

[67] T. Sjostrand, S. Mrenna, and P. Z. Skands, "A Brief Introduction to PYTHIA 8.1," Comput. Phys. Commun. 178 (2008) 852-867, arXiv:0710.3820 [hep-ph].

[68] A. L. Read, "Presentation of search results: The CL(s) technique," J. Phys. G28 (2002) 2693-2704.

[69] A. L. Read, "Modified frequentist analysis of search results (The CL(s) method)," in Workshop on confidence limits, CERN, Geneva, Switzerland, 17-18 Jan 2000: Proceedings. http://weblib. cern. ch/abstract?CERN-OPEN-2000-205.

[70] https://lapth.cnrs.fr/micromegas. 